\title{
CAPACIDADE DE ENRAIZAMENTO DE ESTACAS SEMILENHOSAS E LENHOSAS DE CULTIVARES DE PESSEGUEIRO TRATADAS COM AIB ${ }^{1}$
}

\author{
ADRIANA PEREIRA DE OLIVEIRA², ALEXANDRE AUGUSTO NIENOW ${ }^{3}$, EUNICE DE OLIVEIRA CALVETE $^{4}$
}

\begin{abstract}
RESUMO - Considerando a necessidade de buscar técnicas mais eficientes na produção de mudas de pessegueiro, realizou-se o presente trabalho, com o objetivo de verificar o potencial de enraizamento de estacas, semilenhosas e lenhosas, de seis cultivares de pessegueiro (BR 3, Chula, Coral, Eldorado, Marli e Sinuelo), tratadas com ácido indolbutírico (AIB) nas concentrações de 1500 e $3000 \mathrm{mg} . \mathrm{L}^{-1}$ (imersão da base da estaca por 5 segundos), comparadas com uma testemunha (sem AIB). Foram utilizadas estacas com $20 \mathrm{~cm}$ de comprimento, coletadas em dezembro/2000 (semilenhosas com 4 folhas) e abril/2001 (lenhosas sem folhas). A estaquia foi realizada em tubetes plásticos, contendo casca de arroz carbonizada e mantidas durante 90 dias em estufa equipada com nebulização intermitente. O uso do AIB aumentou, para todas as cultivares e tipos de estacas, a sobrevivência e a porcentagem de enraizamento. A concentração de $1500 \mathrm{mg} . \mathrm{L}^{-1}$ foi considerada suficiente, proporcionando enraizamento entre 65,3 $\%$ a $97,2 \%$ (estacas vivas + mortas) e $27,9 \%$ a $88,9 \%$ de estacas vivas enraizadas. Nesta dose, considerando apenas estacas vivas, as maiores porcentagens de enraizamento foram verificadas nas cvs. Chula, Sinuelo e Marli, com estacas semilenhosas, e nas cvs. Chula e Eldorado, utilizando estacas lenhosas. Por permitir maior facilidade de manuseio, devido à ausência de folhas e menor taxa de mortalidade, as estacas lenhosas, coletadas em abril, são mais indicadas para a produção de mudas.
\end{abstract}

Termos de indexação: Prunus persica, pêssego, estaquia, propagação vegetativa.

\section{ROOTING POTENTIAL CAPACITY OF PEACH TREE CULTIVARS OF SEMI-HARDWOOD AND HARDWOOD CUTTINGS TREATED WITH IBA}

\begin{abstract}
Considering the necessity of search more efficacious technique in the peach plant propagation, this work was realized with the objective to determine six peach tree cultivars of the semi-hardwood and hardwood cuttings rooting potential (BR 3, Chula, Coral, Eldorado, Marli and Sinuelo), treated with IBA at rates of 1500 and $3000 \mathrm{mg} . \mathrm{L}^{-1}$ (immersed 5 seconds), compared with a control (without IBA). Were used cuttings with 20 $\mathrm{cm}$ length, collected in December 2000 (semi-hardwood with 4 leaves) and in April 2001 (hardwood without leaf). The cuttings were done in plastic tubets, containing carbonized rice husk, during 90 days in a greenhouse with intermittent mist. The indol-butiric acid increased, for all cultivars and types of cuttings, the survival and the rooting percentage. The $1500 \mathrm{mg} \cdot \mathrm{L}^{-1}$ was considered the appropriate concentration, providing rooting between $65.3 \%$ to $97.2 \%$ (live + dead cuttings) and $27.9 \%$ to $88.9 \%$ (live rooting cuttings). In this concentration, considering only live cuttings, the most rotting percentage was obtained in Chula, Sinuelo and Marli cultivars with semi-hardwood cutting and Chula and Eldorado cultivars with hardwood cuttings. The hardwood cuttings are more indicated for plant propagation.
\end{abstract}

Index Terms: Prunus persica, peach, cutting, vegetative propagation.

\section{INTRODUÇÃO}

A propagação do pessegueiro pelo método da estaquia pode ser mais vantajosa que a tradicional enxertia pela facilidade de execução e redução do tempo necessário à produção da muda. Pode também evitar alguns inconvenientes da enxertia, como a incompatibilidade e a variabilidade genética dos porta-enxertos. No entanto, algumas cultivares-copa são sucetíveis a nematóides de galhos, o que se torna uma desvantagem. A estaquia pode ser empregada tanto na produção de cultivares-copa, como de porta-enxertos adaptados às condições adversas de solo, tolerantes a pragas e moléstias ou compatíveis com as principais cultivares-copa. A viabilidade da técnica tem aumentado com a utilização de alguns procedimentos, como o uso exógeno de fitorreguladores e a observância das melhores épocas de realização (Fachinello et al., 1995). Contudo, o potencial genético de cada cultivar exerce influência no enraizamento, variando o comportamento e os requisitos para que esse ocorra (Trevisan, 2000).

Em espécies de difícil enraizamento, a deficiência pode estar no baixo teor de auxina. Nesse caso, é necessária uma complementação com um fitorregulador (Hinojosa, 2000), como o AIB, considerado o mais eficiente para essa finalidade (Chauhan e Maheshwari, 1970; Hartmann e Kester, 1990). A concentração ótima, porém, é variável de acordo com a espécie, cultivar e tipo de estaca utilizada. De acordo com Finardi (1998), para o enraizamento de qualquer tipo de estaca de pessegueiro, são necessários $2000 \mathrm{mg} . \mathrm{L}^{-1}$ de AIB. Alguns autores, no entanto, observaram a necessidade de concentrações diferenciadas e de variações de comportamento entre cultivares.

Shaltout et al. (1998) observaram que a concentração de 4000
mg.L ${ }^{-1}$ de AIB foi a que proporcionou maior porcentagem de enraizamento $(72,5 \%)$ de estacas lenhosas do pessegueiro cv. Nemaguard. Nachtigal (1999) obteve 99,8 \% de enraizamento em estacas herbáceas do pessegueiro cv. Okinawa, utilizando $2000 \mathrm{mg} . \mathrm{L}^{-1}$ de AIB. Biasi et al. (2000), avaliando o enraizamento de estacas semilenhosas, com folhas, de cultivares de pessegueiro e ameixeira, verificaram que, na cv. Ouro, a maior porcentagem de enraizamento (91,2\%) ocorreu com $1475 \mathrm{mg} . \mathrm{L}^{-1} \mathrm{e}$, em 'Coral' e 'Sun Red', valores de $83,7 \%$ e $75 \%$, respectivamente, foram obtidos com 2000 mg.L $\mathrm{L}^{-1}$. Trevisan et al. (2000), avaliando estacas semilenhosas, com folhas, de nove cultivares de pessegueiro tratadas com $3000 \mathrm{mg}^{-\mathrm{L}^{-1}}$ de AIB, obtiveram maior percentual de enraizamento em 'Premier' (58,4\%), 'Chiripá' (50,8\%), 'Pialo' (50\%), 'Chimarrita' (50\%), 'Planalto' (41,5\%), 'Maciel' $(38,8 \%)$ e 'Sinuelo' (29,7\%), sendo que 'Flordaprince' (21,72\%) e 'Peach' $(7,24 \%)$ apresentaram os piores resultados.

A presença de folhas e gemas nas estacas herbáceas e semilenhosas mostra-se de grande influência no enraizamento, já que são produtoras de auxinas, co-fatores de enraizamento (Paiva e Gomes, 1995) e carboidratos necessários à manutenção celular (Hartmann e Kester, 1990).

Este trabalho objetivou verificar a capacidade de enraizamento de estacas semilenhosas e lenhosas, bem como determinar a concentração mais adequada do fitorregulador ácido indolbutírico, em seis cultivares de pessegueiro, recomendadas ao plantio no Rio Grande do Sul, conforme Nienow e Licodiedoff (1996).

\section{MATERIALEMÉTODOS}

O trabalho foi conduzido na Faculdade de Agronomia e Medicina Veterinária da Universidade de Passo Fundo, em Passo Fundo-RS,

\footnotetext{
${ }^{1}$ (Trabalho 169/2002). Recebido: 24/10/2002. Aceito para publicação: 11/07/2003. Parte da dissertação de Mestrado em Agronomia da primeira autora, Área de Concentração em Produção Vegetal, da Faculdade da Agronomia e Medicina Veterinária (FAMV) da Universidade de Passo Fundo (UPF).

${ }^{2}$ Bióloga, Mestre em Agronomia, ex-bolsista Capes, e-mail: adrperoliv@ bol.com.br

${ }^{3}$ Eng. Agr., Dr., orientador, Prof. de Fruticultura, Silvicultura e Horticultura da FAMV, da Universidade de Passo Fundo-RS, e-mail: alexandre@upf.br

${ }^{4}$ Eng $^{\mathrm{a}} \mathrm{Agr}^{\mathrm{a}}$., Dr ${ }^{\mathrm{a}}$., Prof ${ }^{\mathrm{a}}$ de Olericultura e Horticultura da Faculdade de Agronomia e Medicina Veterinária da Universidade de Passo Fundo-RS, e-mail: calveteu@ upf.tche.br
} 
em estufa de polietileno com $50 \%$ de sombreamento. O sistema de irrigação, do tipo nebulização intermitente, era acionado em intervalos de 10 minutos, com duração de 5 segundos para as estacas lenhosas e de 10 segundos para as semilenhosas.

As plantas-matrizes, com 7 anos de idade, pertencentes ao pomar do Centro de Extensão e Pesquisa Agronômica - Cepagro, da UPF, foram submetidas à poda de renovação em agosto de 2000, com o objetivo de estimular a emissão de maior número de brotações.

Foram utilizadas estacas subapicais, com $0,4 \mathrm{~cm}$ de diâmetro e $20 \mathrm{~cm}$ de comprimento, coletadas em dezembro de 2000 (semilenhosas com 4 folhas) e abril de 2001 (lenhosas sem folhas), de seis cultivares de pessegueiro (BR 3, Chula, Coral, Eldorado, Marli e Sinuelo) tratadas com ácido indolbutírico (AIB), nas concentrações de 1500 e 3000 mg.L $\mathrm{L}^{-1}$ (imersão da base da estaca por 5 segundos), comparadas com uma testemunha (imersão em água).

O delineamento experimental utilizado foi o inteiramente casualizado, com os tratamentos arranjados em parcelas subdivididas, considerando como parcela principal o tipo de estaca e como subparcelas cultivares x concentrações de AIB. Cada parcela foi constituída de 18 estacas, com quatro repetições.

As estacas foram tratadas com hipoclorito de sódio (Q'Boa $\left.{ }^{\circledR}\right)$ a $1 \%$ e fungicida benomyil (Benlate $500^{\circledR}$ ) a $0,05 \%$ de i.a. Duas lesões longitudinais $(3 \mathrm{~cm})$ foram feitas, com canivete, na base das mesmas e, após o tratamento com AIB, realizado o enterrio de $1 / 3$ de sua porção em tubetes plásticos $\left(100 \mathrm{~cm}^{3}\right)$ contendo casca de arroz carbonizada.

Durante a condução do experimento, foram realizadas pulverizações quinzenais, nas estacas semilenhosas, com o fungicida benomyl (Benlate $500^{\circledR}$ ) a $0,03 \%$ de i.a., acrescido do espalhante adesivo Tween $^{\circledast}$ (4 gotas. $\left.\mathrm{L}^{-1}\right)$, e, nas estacas lenhosas, com o fungicida Metalaxyl/ Mancozeb (Ridomil Mancozeb BR ${ }^{\circledR}$ ), a 0,024 \% e 0,192\% de i.a., respectivamente. Nas estacas semilenhosas, foram realizadas adubações foliares quinzenais, utilizando o produto comercial Niphokam $108^{\circledR}$, na dose recomendada para frutíferas $\left(1,5 \mathrm{~mL} . \mathrm{L}^{-1}\right)$.

Neste trabalho, são apresentados os resultados obtidos de porcentagem de estacas enraizadas, de estacas vivas enraizadas e de estacas mortas enraizadas, avaliadas 90 dias após a permanência das mesmas na estufa.

Verificada a necessidade de avaliar o tempo para o início do enraizamento, foi instalada, para as estacas lenhosas, uma parcelacontrole constituída de 12 estacas por tratamento. A primeira avaliação ocorreu 21 dias após o enterrio e, posteriormente, a cada 8 dias, até que pelo menos uma estaca apresentasse raiz, examinando três estacas por tratamento a cada verificação. Não constatada a presença de raiz, eram recolocadas no substrato e, na avaliação seguinte, outras três estacas eram analisadas. Apenas os tratamentos que exigiram maior termpo para formar raiz foi necessário, após a avaliação de todas as estacas, utilizar as primeiras para dar seqüência à avaliação.

Os dados coletados foram submetidos à análise de variância pelo teste $\mathrm{F}$, conforme o esquema de parcelas subdivididas. As diferenças entre médias foram comparadas pelo teste de Tukey $(\mathrm{P} \leq 0,05)$.

\section{RESULTADOS E DISCUSSÃO}

O tratamento com AIB proporcionou, nas cultivares em estudo, maior porcentagem de enraizamento e de sobrevivência de estacas, tanto semilenhosas como lenhosas.

A porcentagem total de estacas enraizadas (vivas + mortas), tratadas com AIB, variou de 59,7\% a 98,6\% (Tabela 1). Entre as concentrações de 1500 e $3000 \mathrm{mg} . \mathrm{L}^{-1}$, não houve diferença significativa, exceto em estacas lenhosas da cv. Eldorado, que diminuíram a porcentagem de enraizamento com a concentração mais elevada, evidenciando uma provável fitotoxidade. Na ausência do AIB, onde se observa o real potencial genético de enraizamento, as cvs. Marli, Chula e BR 3 mostraram-se menos dependentes do uso do AIB do que as demais cultivares.

As porcentagens de enraizamento obtidas com o uso do AIB, em geral acima de $70 \%$, são consideradas satisfatórias, concordando e até superando as obtidas por autores que trabalharam com outras cultivares, os quais também constataram a influência benéfica do AIB no enraizamento, como Chauhan e Maheshwari (1970) e Shaltout et al. (1998).

$\mathrm{Na}$ comparação dos dois tipos de estacas, tratadas com AIB, em geral as estacas semilenhosas apresentaram maior percentual de enraizamento que as lenhosas. Apenas a cv. Eldorado, com $1500 \mathrm{mg} . \mathrm{L}^{-1}$, proporcionou maior porcentagem total de estacas lenhosas enraizadas, em relação às semilenhosas.

A menor lignificação dos tecidos e a presença de folhas nas estacas semilenhosas devem ter contribuído para o maior enraizamento. Além disso, a poda de renovação, realizada em agosto nas plantasmatrizes, evitou a frutificação, o que pode ter possibilitado o maior acúmulo de reservas nas estacas.

De acordo com Hartmann e Kester (1990), Paiva e Gomes (1995) e Finardi (1998) a menor lignificação dos tecidos das estacas semilenhosas facilita a passagem das raízes formadas no periciclo. Tofanelli et al. (1997) também verificaram maiores percentuais de enraizamento em estacas semilenhosas. Respostas diferenciadas em função da cultivar foram observadas por Tofanelli et al. (1998), quando obtiveram maior porcentagem de enraizamento em estacas lenhosas da cv. Diamante $(55 \%)$ e em estacas semilenhosas das cvs. Pérola de Mairinque (70\%) e Tropical (69 \%), tratadas com 3000 mg.L.-1 de AIB.

Para as estacas lenhosas, no entanto, o enraizamento provavelmente tenha sido prejudicado pela ausência de folhas e pela floração das estacas, que ocorreu cerca de 30 dias após a estaquia. De acordo com Hartmann e Kester (1990) e Fachinello et al. (1995), há uma competição por reservas entre a regeneração vegetativa e a floração.

A porcentagem de enraizamento obtida em estacas semilenhosas da cv. Coral $(88,8 \%$ a 90,2 \%), tratadas com AIB, está próxima da obtida por Biasi et al. (2000) com a mesma cultivar (83,7 \%). Para a cv. Sinuelo, no entanto, a taxa de enraizamento de estacas semilenhosas tratadas com $3000 \mathrm{mg} . \mathrm{L}^{-1}$ de AIB $(87,5 \%)$ foi superior ao obtido por Trevisan et al. (2000), de 29,7\%

Quanto à porcentagem de estacas vivas enraizadas (Tabela 2), apenas 'BR 3', utilizando estacas semilenhosas, não respondeu ao trata-

TABELA 1 - Porcentagem total de estacas enraizadas, semilenhosas e lenhosas, de seis cultivares de pessegueiro, tratadas ou não com AIB - Passo Fundo-RS, 2001

\begin{tabular}{|c|c|c|c|c|c|c|}
\hline \multicolumn{7}{|c|}{ Total de estacas enraizadas (\%) } \\
\hline \multirow{4}{*}{ Cultivares } & \multicolumn{6}{|c|}{$\begin{array}{l}\text { Tipo de estaca } \\
\end{array}$} \\
\hline & \multicolumn{3}{|c|}{ Semilenhosa } & \multicolumn{3}{|c|}{ Lenhosa } \\
\hline & \multicolumn{3}{|c|}{ AIB (mg.L $\left.{ }^{-1}\right)$} & \multicolumn{3}{|c|}{ AIB (mg.L $\left.\mathrm{L}^{-1}\right)$} \\
\hline & 0 & 1500 & 3000 & 0 & 1500 & 3000 \\
\hline BR-3 & B 36,1 b & A 93,0* a & A $94,4^{*}$ a & B 30,6 ab & A 65,3 b & A $66,7 \mathrm{a}$ \\
\hline Chula & B 20,8 bc & A 97,2 a & A $98,6 *$ a & B 56,9* a & A 97,2 a & $\mathrm{AB} 72,2 \mathrm{a}$ \\
\hline Coral & B 8,3 c & A $90,2 *$ a & A $88,8 * a b$ & B 6,9 b & A 75,0 ab & A $\quad 59,7$ a \\
\hline Eldorado & B $0,0 \quad c$ & A 75,0 a & A 70,8 b & C 9,7 b & A $93,1 * a$ & $62,5 \mathrm{a}$ \\
\hline Marli & B $59,7 * a$ & A $94,4 * a$ & A 91,6 ab & B 44,4 a & A 80,6 ab & 84,7 a \\
\hline Sinuelo & B 5,5 c & A $91,6^{*}$ a & A 87,5 ab & B 8,3 b & A 76,4 ab & $77,8 \mathrm{a}$ \\
\hline
\end{tabular}

Médias antecedidas de mesma letra maiúscula na horizontal e seguidas de mesma letra minúscula linha não diferem entre si, pelo teste de Tukey (P = 0,05).

* Significativamente superior, na comparação entre estacas semilenhosas e lenhosas, para a mesma concentração de AIB $(\mathrm{P}=0,05)$. 
mento com AIB, apresentando baixas porcentagens de sobrevivência, decorrentes, possivelmente, de fatores endógenos do genótipo. No entanto, o uso de estacas lenhosas dessa cultivar, tratadas com $3000 \mathrm{mg} . \mathrm{L}$ ${ }^{1}$, possibilitou um aumento na porcentagem de estacas vivas enraizadas.

As cvs. Chula, Sinuelo, Marli e Coral destacaram-se por proporcionar maior porcentagem de estacas vivas enraizadas, quando utilizadas estacas semilenhosas tratadas com AIB. Em estacas lenhosas tratadas com AIB, mais uma vez, destacou-se a cv. Chula, além de Eldorado, Marli e Sinuelo. Diferenças entre cultivares na porcentagem de sobrevivência foram também relatadas por Trevisan et al. (2000).

$\mathrm{O}$ efeito do tipo de estaca sobre a porcentagem de estacas vivas enraizadas variou entre as cultivares, para uma mesma concentração de AIB. Assim, sem tratamento com fitorregulador, as estacas lenhosas de 'Chula' e 'Marli' proporcionaram maior porcentagem em relação às semilenhosas. Com $1500 \mathrm{mg} . \mathrm{L}^{-1} \mathrm{de} \mathrm{AIB}$, estacas lenhosas de 'Eldorado' apresentaram maior sobrevivência, com 'Sinuelo' comportando-se de forma inversa. Com $3000 \mathrm{mg} . \mathrm{L}^{-1}$ de AIB, a maior porcentagem de estacas vivas enraizadas ocorreu nas semilenhosas de 'Chula' e lenhosas de 'Marli', 'Eldorado' e 'BR 3'.

A obtenção de maior porcentagem de estacas vivas enraizadas pelo uso do AIB, provavelmente, deveu-se à melhoria no balanço hormonal das estacas, mas também à redução do tempo necessário para iniciar o enraizamento.

Na parcela instalada para avaliar o tempo necessário para o início do enraizamento, foi constatado que, embora os processos internos de formação da raiz tenham se iniciado antes, com o uso do AIB, a exteriorização das raízes, nas estacas, ocorreu entre 21 e 29 dias para as cvs. Marli, Chula, Coral e BR 3, em 37 dias para Eldorado e 45 dias para Sinuelo. Sem o uso do AIB, apenas as cvs. Coral (52 dias) e Eldorado (75 dias) formaram raízes.

O uso do AIB proporcionou maior porcentagem total de estacas enraizadas (Tabela 1) e redução do tempo necessário para a formação das raízes, mas não impediu, que mesmo já enraizadas, uma porcen- tagem significativa morresse (Tabela 3). Para as estacas semilenhosas das cvs. Chula e Marli, e lenhosas de Chula e BR 3, não houve influência do uso ou não do fitorregulador sobre a porcentagem de estacas mortas enraizadas. Estacas lenhosas das cvs. Coral, Sinuelo, Marli e Eldorado, tratadas com 1500 mg. $\mathrm{L}^{-1}$ de AIB, apresentaram maior mortalidade, não diferindo Coral e Eldorado do tratamento com $3000 \mathrm{mg} . \mathrm{L}^{-1}$.

A maior mortalidade de estacas semilenhosas enraizadas, na ausência do AIB, ocorreu nas cvs. Marli, BR 3 e Chula, que haviam apresentado maior percentual total de enraizamento (Tabela 1).

Na comparação entre tipos de estacas, em geral, a maior porcentagem de estacas mortas enraizadas ocorreu nas semilenhosas. No período de enraizamento dessas estacas, a temperatura média no interior da estufa manteve-se, aproximadamente, $10^{\circ} \mathrm{C}$ acima da temperatura verificada com as estacas lenhosas. Esse fator, aliado à menor lignificação dos tecidos e à presença de folhas, possivelmente aumentou a transpiração, causando a desidratação e a rápida degradação das reservas da estaca, apesar da nebulização intermitente. Por outro lado, as temperaturas mais baixas, nas estacas lenhosas, podem ter reduzido a atividade enzimática e, com isso, retardado o início do enraizamento, conforme relataram Fachinello et al. (1995) e Hartmann e Kester (1990).

De acordo com Nachtigal (1999), a morte de estacas após a formação das raízes é, provavelmente, consequiência da manutenção das mesmas na câmara de nebulização por um período maior do que o necessário ao enraizamento, causando a morte das raízes por excesso de umidade. Para Trevisan et al. (2000), a mortalidade das estacas também pode estar relacionada ao potencial genético de cada cultivar. Pode ser considerado, ainda, que, uma vez enraizadas, a ausência de nutrientes no substrato possa ter se constituído em um fator de mortalidade das estacas.

Embora em todas as épocas testadas seja possível reproduzir o pessegueiro por estaquia, o uso de estacas lenhosas, coletadas em abril, se mostra mais indicado, por permitir maior facilidade de manuseio e preparação das estacas, devido a ausência de folhas, e a menor taxa de mortalidade.

TABELA 2 - Porcentagem de estacas vivas enraizadas, semilenhosas e lenhosas, de seis cultivares de pessegueiro, tratadas ou não com AIB - Passo Fundo-RS, 2001

\begin{tabular}{|c|c|c|c|c|c|c|}
\hline \multicolumn{7}{|c|}{ Estacas vivas enraizadas (\%) } \\
\hline \multirow{4}{*}{ Cultivares } & \multicolumn{6}{|c|}{ Tipo de estaca } \\
\hline & \multicolumn{3}{|c|}{ Semilenhosa } & \multicolumn{3}{|c|}{ Lenhosa } \\
\hline & \multicolumn{3}{|c|}{ AIB (mg.L $\left.\mathrm{L}^{-1}\right)$} & \multicolumn{3}{|c|}{ AIB (mg. $\left.\mathrm{L}^{-1}\right)$} \\
\hline & 0 & 1500 & 3000 & 0 & 1500 & 3000 \\
\hline BR-3 & A $12,5 \mathrm{ab}$ & A 27,9 c & A 16,7 d & B 13,9 b & $\mathrm{AB} 33,3 \mathrm{~d}$ & A $43,1 * b$ \\
\hline Chula & B $8,3 \mathrm{ab}$ & A 84,7 a & A $81,9 *$ a & B $56,9 *$ a & A 88,9 a & $\mathrm{AB} 68,1 \mathrm{ab}$ \\
\hline Coral & B $0,0 \mathrm{~b}$ & A 40,3 bc & A 49,9 bc & B 5,6 b & A $41,7 \mathrm{~cd}$ & A 50,0 b \\
\hline Eldorado & B $0,0 \mathrm{~b}$ & A 29,6 c & A $37,5 \mathrm{~cd}$ & B $8,3 \quad b$ & A $77,8^{*} \mathrm{ab}$ & A $58,3 * a b$ \\
\hline Marli & B 27,7 a & A $61,1 \quad a b$ & A 52,7 bc & $\mathrm{B} 43,1 * \mathrm{a}$ & $\mathrm{AB} 61,1 \mathrm{bc}$ & A $79,2 *$ a \\
\hline Sinuelo & B $1,4 \mathrm{~b}$ & A $77,8 * a$ & A $65,2 \mathrm{ab}$ & B $6,9 \quad b$ & A $44,4 \mathrm{~cd}$ & A 56,9 ab \\
\hline
\end{tabular}

Médias antecedidas de mesma letra maiúscula na coluna e antecedidas de mesma letra minúscula na linha não diferem entre si, pelo teste de Tukey $(\mathrm{P}=0,05)$.

* Significativamente superior, na comparação entre estacas semilenhosas e lenhosas, para a mesma concentração de AIB $(\mathrm{P}=0,05)$.

TABELA 3 - Porcentagem de estacas mortas enraizadas, semilenhosas e lenhosas, de seis cultivares de pessegueiro, tratadas ou não com AIB - Passo Fundo-RS, 2001

\begin{tabular}{|c|c|c|c|c|c|c|c|}
\hline \multicolumn{8}{|c|}{ Estacas mortas enraizadas $(\%)$} \\
\hline \multirow{4}{*}{ Cultivares } & \multicolumn{7}{|c|}{ Tipo de estaca } \\
\hline & \multicolumn{3}{|c|}{ Semilenhosa } & \multicolumn{4}{|c|}{ Lenhosa } \\
\hline & \multicolumn{3}{|c|}{ AIB (mg.L $\left.{ }^{-1}\right)$} & \multicolumn{4}{|c|}{ AIB (mg.L $\left.{ }^{-1}\right)$} \\
\hline & 0 & 1500 & 3000 & 0 & 1500 & & 3000 \\
\hline BR-3 & B 23,6 ab & A $65,3 * a$ & A $77,8 * a$ & A 16,7 a & A 32,0 a & & $23,6 \mathrm{a}$ \\
\hline Chula & A $12,5 *$ abc & A 12,5 c & A $16,7^{*} \mathrm{c}$ & A 0,0 a & A 8,3 a & & $4,2 \mathrm{c}$ \\
\hline Coral & B 8,3 bc & A $50,0^{*} \mathrm{ab}$ & A $38,9 *$ b & B 1,4 a & A 33,3 a & $\mathrm{AB}$ & $9,7 \mathrm{abc}$ \\
\hline Eldorado & B $0,0 \quad \mathrm{c}$ & A $45,8^{*} a b$ & A $33,3 *$ bc & B $1,4 \mathrm{a}$ & A 15,3 a & $\mathrm{AB}$ & $4,2 \mathrm{c}$ \\
\hline Marli & A $32,0 *$ a & A $33,3 *$ bc & A $38,9 * b$ & $1,4 \mathrm{a}$ & A 19,4 a & B & $5,6 \mathrm{bc}$ \\
\hline Sinuelo & B 4,2 bc & $\mathrm{AB} 13,9 \mathrm{c}$ & A 22,2 bc & C $1,4 \mathrm{a}$ & A $31,9 *$ a & $\mathrm{B}$ & $20,8 \mathrm{ab}$ \\
\hline
\end{tabular}

Médias seguidas de mesma letra maiúscula na coluna e antecedidas de mesma letra maiúscula na linha não diferem entre si, pelo teste de Tukey (P = 0,05).

* Significativamente superior, na comparação entre estacas semilenhosas e lenhosas, para a mesma concentração de AIB $(\mathrm{P}=0,05)$. 


\section{CONCLUSÕES}

1) O ácido indolbutírico aumenta, para todas as cultivares e tipos de estacas, a sobrevivência e a porcentagem de enraizamento.

2) A dose de $1500 \mathrm{mg} . \mathrm{L}^{-1}$ de AIB é satisfatória, proporcionando enraizamento entre $65,3 \%$ e $97,2 \%$ (estacas vivas + mortas) e de $27,9 \%$ a $88,9 \%$ de estacas vivas enraizadas.

3) $\mathrm{Na}$ dose de $1500 \mathrm{mg} \cdot \mathrm{L}^{-1}$, considerando apenas as estacas vivas, as maiores porcentagens de enraizamento foram verificadas nas cvs. Chula, Sinuelo e Marli, com estacas semilenhosas, e nas cvs. Chula e Eldorado, utilizando estacas lenhosas.

4) Por permitir maior facilidade de manuseio, devido à ausência de folhas e à menor taxa de mortalidade, as estacas lenhosas, coletadas em abril, são mais indicadas à produção de mudas.

\section{REFERÊNCIASBIBLIOGRÁFICAS}

BIASI, L. A.; STOLTE, R. E.; SILVA, M. F. da. Estaquia de ramos semilenhosos de pessegueiro e nectarineira. Revista Brasileira de Fruticultura, Jaboticabal, v. 22, n. 3, p. 421-425, 2000.

CHAUHAN, K. S.; MAHESHWARI, L. D. Effects of certain plant growth regulators, seasons and types of cutting on root initiation and vegetative growth in stem cuttings of peach variety Sharbati. Indian Journal of Horticulture, Bangalore, v. 27, n. 3/4, p. 136-140, 1970.

FACHINELLO, J. C.; HOFFMANN, A.; NACHTIGAL, J. C.; KERSTEN, E.; FORTES, G. R. de L. Propagação de plantas frutíferas de clima temperado. 2. ed. Pelotas: Ufpel, 1995. 178p.

FINARDI, N. L. Métodos de propagação e descrição de porta-enxertos. In: MEDEIROS, C. A. B.; RASEIRA, M. do C. B. (Ed.). A cultura do pessegueiro. Brasília: Embrapa-SPI; Pelotas: Embrapa-CPACT, 1998. p. $100-129$.

HARTMANN, H. T.; KESTER, D. E. Propagacion de plantas: principios y practicas. 4. ed. México: Continental, 1990.760p.
HINOJOSA, G. F. Auxinas. In: CID, L. P. B. (Ed.) Introdução aos hormônios vegetais. Brasília: Embrapa Recursos Genéticos e Biotecnologia, 2000. p. 15-53.

NACHTIGAL, J. C. Obtenção de porta-enxertos 'Okinawa' e de mudas de pessegueiro (Prunus persica (L.) Batsch) utilizando métodos de propagação vegetativa. 1999. 165f. Tese (Doutorado em Produção Vegetal) - Faculdade de Ciências Agrárias e Veterinárias, Universidade Estadual Paulista, Jaboticabal-SP, 1999.

NIENOW, A. A.; LICODIEDOFF, M. C. Comportamento fenológico e produtivo de cultivares de pessegueiro e nectarineira no Planalto Médio do Rio Grande do Sul. Revista Brasileira de Fruticultura, Cruz das Almas, v. 18, n. 2, p. 201-208, 1996.

PAIVA, H. N. de; GOMES, J. M. Propagação vegetativa de espécies florestais. Viçosa: UFV, 1995. 40p.

SHALTOUT, A. D.; SALAMA, M.; EL-WAKEEL, H. F.; AZIZ, M. B. A.; ISMAIL, O. M. Propagation of Nemaguard peach by stem hardwood cuttings and layerings. Annals of Agricultural Science, Cairo, v. 3, p. 865-879, 1998.

TOFANELLI, M. B. D.; CHALFUN, N. N. J.; HOFFMANN, A.; ANTUNES, L. E. C. Enraizamento de estacas lenhosas e semilenhosas de cultivares copa de pessegueiro em diferentes concentrações de ácido indolbutírico. Revista Brasileira de Fruticultura, Cruz das Almas, v. 19, n. 2, p. 259-263, 1997.

TOFANELLI, M. B. D.; CHALFUN, N. N. J.; HOFFMANN,A.; CHALFUN JÚNIOR, A.; NORBERTO, P. M. et al. Capacidade de enraizamento de estacas lenhosas e semilenhosas de cultivares de pessegueiro. In: CONGRESSO BRASILEIRO DE FRUTICULTURA, 15, 1998, Poços de Caldas. Resumos... Lavras: Ufla, 1998. p. 684.

TREVISAN, R.; SCHWARTZ, E.; KERSTEN, E. Capacidade de enraizamento de estacas de ramos de pessegueiro (Prunus persica (L.) Batsch) de diferentes cultivares. Revista Científica Rural, Bagé, v. 5, n. 1, p. 29-33, 2000. 